\title{
Wireless Transmission in Tunnels
}

\author{
Samir F. Mahmoud \\ Kuwait University \\ Kuwait
}

\section{Introduction}

Study of electromagnetic wave propagation within tunnels was driven in the early seventies by the need for communication among workers in mine tunnels. Such tunnels are found in the form of a grid of crossing tunnels running for several kilometers, which called for reliable means of communication. Since then a great number of contributions have appeared in the open literature studying the mechanisms of communication in tunnels. Much experimental and theoretical work was done in USA and Europe concerning the development of wireless and wire communication in tunnels. A typical straight tunnel with cross sectional linear dimensions of few meters can act as a waveguide to electromagnetic waves at UHF and the upper VHF bands; i.e. at wavelengths in the range of a fraction of a meter to few meters. In this range, a tunnel is wide enough to support free propagation of electromagnetic waves, hence provides communication over ranges of up to several kilometers. At those high frequencies, the tunnel walls act as good dielectric with small loss tangent. For example at a frequency of $1000 \mathrm{MHz}$, the rocky wall with $10^{-2}$ Siemens $/ \mathrm{m}$ conductivity and relative permittivity of about 10 has a loss tangent equal to 0.018 . So the electromagnetic wave losses will be caused mainly by radiation or refraction through the walls with little or negligible ohmic losses as deduced by Glaser $(1967,1969)$.

Goddard (1973) performed some experiments on UHF and VHF wave propagation in mine tunnels in USA. His work was presented in the Thru-Earth Workshop held in Golden, Colorado in August 1973. Goddard's experimental results show that small attenuation is attained in the UHF band. He also measured wave losses around corners and detected high coupling loss between crossing tunnels. The theory of mode propagation of electromagnetic waves in tunnels of rectangular cross section was reported by Emslie et al (1973) in the same workshop and later in (1975) in the IEEE, AP journal. Their presentation extended the theory given earlier by (Marcatelli and Schmeltzer, 1964) and have shown, among other things, that the modal attenuation decreases with the applied frequency squared. Chiba et al. (1978) presented experimental results on the attenuation in a tunnel with a cross section close to circular except for a flat base. They have shown that the measured attenuation closely match the theoretical attenuation of the dominant modes in a circular tunnel of equal cross section. Mahmoud and Wait (1974a) applied geometrical ray theory to obtain the fields of a dipole source in a tunnel with rectangular cross section of linear dimensions of several wavelengths. The same authors (Mahmoud and Wait, 1974b) studied the attenuation in a 
curved rectangular tunnel showing a considerable increase in attenuation due to the curvature.

Recent advances on wireless communication, in general, have revived interest in continued studies of free electromagnetic transmission in tunnels. Notable contributions have been made by Donald Dudley in USA and Pierre Degauque in France and their research teams.

In the next sections, we review the theoretical early and recent studies done on communication within tunnels. We start by reviewing the mode theory of propagation in a straight tunnel model with a circular cross-section. We obtain the propagation parameters of the important lower order modes in closed forms. Such modes dominate the total field at sufficiently distant points from the source. We follow by reviewing mode propagation in tunnels with rectangular cross section. Tunnels with arched cross sections are then treated as perturbed tunnel shapes using the perturbation theory to characterize their dominant modes. We also review studies made on wave propagation in curved tunnels and propagation around corners. We conclude by comparisons between theory and available experimental results.

\section{Tunnel wall Characterization}

Before treating modal propagation in tunnels, it will prove useful to characterize the tunnel wall as constant impedance surfaces for the dominant modes of propagation. This is covered in detail in [Mahmoud, 1991, chapter 3] for planar and cylindrical guides. Here we give a simplified argument for adopting the concept of constant impedance wall. By this term, we mean that the surface impedance of the wall is almost independent of the angle of wave incidence onto the wall. So, let us consider a planar surface separating the tunnel interior (air) from the wall medium with relative permittivity $\varepsilon_{\mathrm{r}}$ and permeability $\mu_{0}$ where $\varepsilon_{\mathrm{r}}$ is usually $>>1$. At a given applied angular frequency $\omega$, the bulk wavenumber in air is $k_{0}=\omega \sqrt{\mu_{0} \varepsilon_{0}}$ and in the wall is $k=k_{0} \sqrt{\varepsilon_{r}}>k_{0}$. We may define three right handed mutually orthogonal unit vectors $\hat{n}, \hat{t}, \hat{z}$ where $\hat{t}$ and $\hat{z}$ are tangential to the air-wall interface and $\hat{n}$ is normal to the interface and is directed into the wall. Now consider a wave that travels along the interface with dependence $\exp (j \omega t-j \beta z)$ in both the air and wall. For modes with $\beta<k_{0}$, one has $k_{0}^{2}-\beta^{2}<k_{0}^{2}$ and definitely $k_{0}^{2}-\beta^{2}<<k^{2}$. Hence the transverse wavenumber in the wall (along $\hat{n}$ ) $k_{n}=\sqrt{k^{2}-\beta^{2}}$ can be well approximated by the $k_{n}=\sqrt{k^{2}-k_{0}^{2}}$ which is independent of $\beta$. If the wave is $\mathrm{TE}_{\mathrm{n}}$ polarized, i.e. having zero $E_{n}$ and nonzero $H_{n}$, the surface impedance of the wall is given by:

$$
Z_{s}=E_{t} / H_{z}=\omega \mu_{0} / \sqrt{k^{2}-k_{0}^{2}}=\eta_{0} / \sqrt{\varepsilon_{r}-1}
$$

Similarly if the wave is $\mathrm{TM}_{\mathrm{n}}$ polarized, the surface admittance of the wall is

$$
Y_{S}=H_{t} / E_{z}=-\omega \varepsilon / \sqrt{k^{2}-k_{0}^{2}}=\varepsilon_{r} / \eta_{0} \sqrt{\varepsilon_{r}-1}
$$

where $\eta_{0}$ is the wave impedance in $\operatorname{air}(=120 \pi \Omega)$. 
The $Z_{s}$ and $Y_{s}$ in (1-2) are the constant impedance/admittance of the wall. When the air-wall interface is of cylindrical shape, the same $Z_{s}$ and $Y_{s}$ apply provided that $\left(k^{2}-k_{0}^{2}\right)^{1 / 2} a \gg 1$ ([Mahmoud, 1991), where $a$ is the cylinder radius. This condition is satisfied in most tunnels at the operating frequencies.

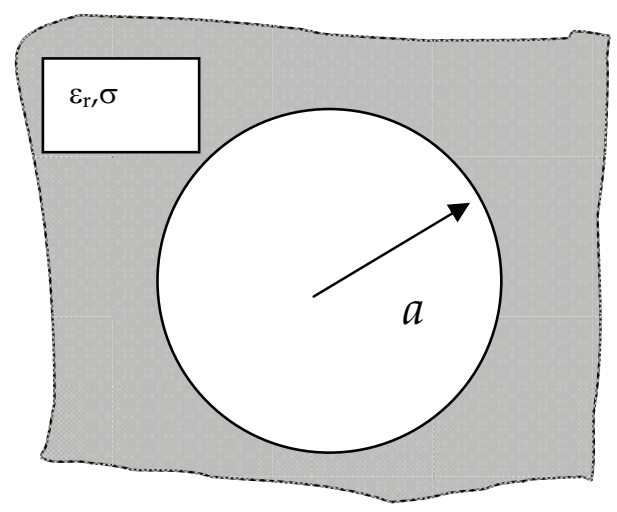

Fig. 1. A circular cylindrical tunnel of radius ' $a$ ' in a host medium of relative permittivity $\varepsilon_{\mathrm{r}}$ and conductivity ' $\sigma$ '.

\section{Modal propagation in a tunnel with a circular cross Section}

An empty tunnel with a circular cross section is depicted in Figure 1. The surrounding medium has a relative permittivity $\varepsilon_{r}$ (assumed $\gg 1$ ) and conductivity $\sigma \mathrm{S} / \mathrm{m}$. The circular shape allows for rigorous treatment of the free propagating modes. The modal equation for the various propagating modes was derived by Stratton [1941] and solved approximately for the modal phase and attenuation constants of the low order modes in (Marcatili and Shmeltzer, 1964), (Glasier, 1969) and more recently by Mahmoud (1991). In the following, we derive modal solutions that are quite accurate as long as the tunnel diameter is large relative to the applied wavelength. Under these conditions the tunnel wall is characterized by the constant surface impedance and admittance given in (1-2). Specialized to the circular tunnel, they take the form:

$$
E_{\phi}=\eta_{0} \bar{Z}_{s} H_{z} \quad \text { and } \quad \eta_{0} H_{\phi}=-\bar{Y}_{s} E_{z}
$$

Here $\bar{Z}_{s}$ and $\bar{Y}_{s}$ are normalized impedance and admittance relative to $\eta_{0}$ and $\eta_{0}{ }^{-1}$ respectively. Explicitly:

$$
\begin{gathered}
\bar{Z}_{s}=1 / \sqrt{\varepsilon_{r}-1-i \sigma / \omega \varepsilon_{0}}, \\
\bar{Y}_{s}=\left(\varepsilon_{r}-i \sigma / \omega \varepsilon_{0}\right) / \sqrt{\varepsilon_{r}-1-i \sigma / \omega \varepsilon_{0}}
\end{gathered}
$$


Because of the imperfectly reflecting walls, the allowed modes are generally hybrid with nonzero longitudinal field components $E_{z}$ and $H_{z}$ (Mahmoud, 1991, sec. 3.4). Thus:

$$
\begin{gathered}
E_{z}=j J_{n}\left(k_{\rho} \rho\right) \sin n \phi \exp (-j \beta z) \\
\eta_{0} H_{z}=-j \Lambda J_{n}\left(k_{\rho} \rho\right) \cos m \phi \exp (-j \beta z)
\end{gathered}
$$

where $\Lambda$ is the mode hybrid factor, $\beta$ is the mode propagation factor, $J($.$) is the Bessel$ function of first kind, and $n$ is an integer $=0,1,2 \ldots$. The transverse fields $E_{\rho}, E_{\phi}, H_{\rho}$, and $H_{\phi}$ are obtainable in terms of the longitudinal components through well-known relations as given in the Appendix. The boundary conditions at the tunnel wall $\rho=a$ require that $E_{\phi}=\eta_{0} \bar{Z}_{s} H_{z}$ and $\eta_{0} H_{\phi}=-\bar{Y}_{s} E_{z}$. Using (A2) \& A(3) in the Appendix along with (5), these boundary conditions read:

$$
\begin{aligned}
& F(u)+j \bar{Z}_{s} u^{2} / \mathrm{v}=-\beta n / \Lambda k_{0} \\
& F(u)+j \bar{Y}_{s} u^{2} / \mathrm{v}=-\beta n \Lambda / k_{0}
\end{aligned}
$$

where:

$$
\begin{gathered}
u=k_{\rho} a, \mathrm{v}=k_{0} a, \\
\mathrm{v}^{2}-u^{2}=(\beta a)^{2}, \text { and } \\
F_{n}(u)=\frac{u J_{n}^{\prime}(u)}{J_{n}(u)} \equiv n \frac{J_{n-1}(u)-J_{n+1}(u)}{J_{n-1}(u)+J_{n+1}(u)}
\end{gathered}
$$

The prime denotes differentiation with respect to the argument and the last equality stems from identities in (A4).

Equations (6) lead to the modal equation for the propagation factor $\beta$ and the mode hybrid factor $\Lambda$. Namely:

$$
\begin{gathered}
\left(F_{n}(u)+j u^{2} \bar{Y}_{s} / \mathrm{v}\right)\left(F_{n}(u)+j u^{2} \bar{Z}_{s} / \mathrm{v}\right)-n^{2}\left(\beta / k_{0}\right)^{2}=0 \\
\Lambda-\Lambda^{-1}=-j\left(\bar{Y}_{s}-\bar{Z}_{s}\right) u^{2} k_{0} / n \beta \mathrm{v}
\end{gathered}
$$

\subsection{The $\phi$ - symmetric modes $(n=0)$}

Considering first the $\phi$-symmetric modes we set $n=0$ in (8) which then reduces to two equations for the $\mathrm{TM}_{0 \mathrm{~m}}$ and $\mathrm{TE}_{0 \mathrm{~m}}$ modes; namely:

$$
\begin{gathered}
F_{0}(u)=-u J_{1}(u) / J_{0}(u)=-j u^{2} \bar{Y}_{s} / \mathrm{v} \\
F_{0}(u)=-u J_{1}(u) / J_{0}(u)=-j u^{2} \bar{Z}_{s} / \mathrm{v}
\end{gathered}
$$


For the low order modes which are dominant at sufficiently high frequencies, $u / v<<1$, hence the RHS of $(9 a-9 b)$ are also $<<1$ and an approximate solution for the eigenvalue $u$ is:

$$
\begin{aligned}
& u_{0 m} \cong x_{1 m}\left(1+j \bar{Y}_{s} / \mathrm{v}\right) \\
& u_{0 m} \cong x_{1 m}\left(1+j \bar{Z}_{s} / \mathrm{v}\right)
\end{aligned}
$$

for $\mathrm{TM}_{0 \mathrm{~m}}$ and $\mathrm{TE}_{0 \mathrm{~m}}$ respectively. In the above $x_{1 m}$ is the $m$ th root of the Bessel function $\mathrm{J}_{1}$. Note that the second of (8) yields $\Lambda=0\left(\mathrm{H}_{\mathrm{z}}=0\right)$ or infinity $\left(\mathrm{E}_{\mathrm{z}}=0\right)$ as expected for the $\mathrm{TM}_{0}$ and $\mathrm{TE}_{0}$ modes respectively. Since $\beta a=\sqrt{v^{2}-u^{2}}$, we can use (10-11) to get the complex $\beta$ factor. Of particular interest are the modal attenuation rates, which take the forms

$$
\alpha_{0 m}=\frac{x_{1 m}^{2}}{k_{0}^{2} a^{3}} \operatorname{Re}\left[\begin{array}{c}
\bar{Z}_{s} \\
\bar{Y}_{s}
\end{array}\right] \quad \text { Neper } / \mathrm{m}
$$

The $T E_{0}$ modes are associated with $Z_{\mathrm{s}}$ and the $T M_{0}$ modes with $\mathrm{Y}_{\mathrm{s}}$. Since $\bar{Z}_{s}<<\bar{Y}_{s}$ (see (4)), it is clear that the $T E_{0}$ modes have considerably less attenuation than the $T M_{0}$ modes; it is less by almost the factor $\varepsilon_{r}$.

\subsection{Hybrid Modes}

Next we consider the case $n>0$ for the hybrid modes. In the high frequency regime and for the low order modes, we use the following approximations in (8): $\left(\beta / \mathrm{k}_{0}\right)^{2 \sim} 1$, and $u^{2} / \mathrm{v}<<1$. This leads to the approximate solutions:

$$
u_{n m}=x_{n-1, m}\left(1+j\left(\bar{Z}_{s}+\bar{Y}_{s}\right) / 2 \mathrm{v}\right)
$$

and

$$
u_{n m}=x_{n+1, m}\left(1+j\left(\bar{Z}_{s}+\bar{Y}_{s}\right) / 2 \mathrm{v}\right)
$$

The corresponding modes are designated as $H E_{n m}$ and $E H_{n m}$ modes respectively. Investigating the hybrid factor $\Lambda$ (the second of eqn. 8 ), we find that it approaches +1 for $H E$ and -1 for $E H$ modes in the infinite frequency limit. This means that the modes are hybrid balanced modes in this limit. Further discussions on these modes are found in (Mahmoud,1991, Chap.5).

Using (13)-(14) along with the relation $\beta a=\sqrt{v^{2}-u^{2}}$, we obtain the high frequency modal propagation constant. Again, the imaginary part of $\beta$ gives the mode attenuation rate $\alpha(\alpha=-$ $\operatorname{Im}(\beta))$. The approximate modal attenuation factor $\alpha$ for the $\mathrm{HE}_{\mathrm{nm}}$ modes is:

$$
\alpha_{n m}=\frac{x_{n-1, m}^{2}}{2 k_{0}^{2} a^{3}} \operatorname{Re}\left[\bar{Y}_{s}+\bar{Z}_{s}\right] \quad(\text { Neper } / \mathrm{m})
$$

The attenuation rate for the other set of modes; $\mathrm{EH}_{\mathrm{nm}}$ modes, is the same except that the Bessel root $x_{n-1, m}$ is replaced by $x_{n+1, m}$. The above formulae (12 and 15) show that the modal attenuation is inversely proportional to the frequency squared and the radius cubed. Note 
that these Formulae are restricted to the lower order modes of the tunnel at sufficiently high frequencies.

It is clear that the least attenuated mode of the $T E_{0}$ and $T M_{0}$ mode group is the $T E_{01}$ mode, while the least attenuated hybrid mode is the $H E_{11}$ mode. It is interesting to compare the attenuation rates of these two modes; namely the $T E_{01}$ and the $H E_{11}$ mode. Using (12) and (15), we get the ratio

$$
\frac{\alpha_{T E 01}}{\alpha_{H E 11}}=\frac{x_{1,1}^{2}}{x_{0,1}^{2}} \frac{2}{\varepsilon_{r}+1}=\frac{3.832^{2}}{2.405^{2}} \frac{2}{\varepsilon_{r}+1}=\frac{5.078}{\varepsilon_{r}+1}
$$

where we have neglected the earth conductivity $\sigma$ relative to $\omega \varepsilon$. As an example, for typical earth with $\varepsilon_{r}=12$, the above ratio amounts to 0.395 , which means that the $T E_{01}$ mode is the least attenuated mode in a typical circular tunnel.

In order to check the approximate closed forms (12)-(15) for the attenuation factors, we compare them with exact results presented recently by Dudley and Mahmoud [2006] in Table 1. The table lists the attenuation rates of some of the dominant modes in a circular tunnel of 2 meter radius and outer medium having $\varepsilon_{r}=12$ at $1 \mathrm{GHz}$. It is seen that the percentage error is less than $\sim 2.7 \%$ for all the listed modes except the $\mathrm{EH}_{11}$ mode. This mode requires a higher frequency for the approximate attenuation to have a better accuracy.

\begin{tabular}{|l|c|c|c|}
\hline Mode & Exact & Approximate & \% Error \\
\hline $\mathrm{TE}_{01}$ & 1.098 & 1.096 & $0.18 \%$ \\
\hline $\mathrm{TE}_{02}$ & 3.716 & 3.673 & $1.16 \%$ \\
\hline $\mathrm{TE}_{03}$ & 7.937 & 7.724 & $2.68 \%$ \\
\hline $\mathrm{HE}_{11}$ & 2.774 & 2.805 & $1.12 \%$ \\
\hline $\mathrm{HE}_{21}$ & 7.158 & 7.122 & $0.50 \%$ \\
\hline $\mathrm{HE}_{31}$ & 13.12 & 12.79 & $2.52 \%$ \\
\hline $\mathrm{TM}_{01}$ & 13.30 & 13.15 & $1.13 \%$ \\
\hline $\mathrm{EH}_{11}$ & 20.18 & 12.794 & $36.6 \%$ \\
\hline
\end{tabular}

Table 1. Comparison between Exact [Dudley \& Mahmoud,2006] and approximate attenuation rates in $\mathrm{dB} / 100$ meters at $1 \mathrm{GHz}$.

Exercise 1: Verify the approximate attenuation rates in Table 1, Column 3 by using (12) for the $\mathrm{TE}_{0 \mathrm{~m}} / \mathrm{TM}_{0 \mathrm{~m}}$ modes and (15) for the $\mathrm{HE}_{\mathrm{nm}}$ modes.

Exercise 2: Using any root finding software, verify the exact attenuation rates in column 2 of Table 1. To do so, you need to solve the modal equation (8) for the complex roots of $u$. You can use (10),(11) or (13) as initial guess for $\mathrm{u}$ of the $\mathrm{TE}_{0 \mathrm{~m}}, \mathrm{TM}_{0 \mathrm{~m}}$ and $\mathrm{HE}_{\mathrm{nm}}$ modes respectively. Once you get $\mathrm{u}$, the complex $\beta$ is obtained from $\beta a=\sqrt{v^{2}-u^{2}}$. The mode attenuation rate $\alpha$ in Neper $/ m$ is the negative of the imaginary part of $\beta$. To convert to $\mathrm{dB} / 100 \mathrm{~m}$, note that $1 \mathrm{Neper} / \mathrm{m}=868.8 \mathrm{~dB} / 100 \mathrm{~m}$. 


\subsection{Mode Excitation}

In the above we have ordered the modes in ascending order of their attenuation rates. However, the actual level of the modes at a given distance from the source is determined also by their excitation factor, which in turn depends on their field distribution and the source type, location and orientation. The E-field distribution of the $\mathrm{TE}_{01}$ and the $\mathrm{HE}_{11}$ modes, which are the least attenuated modes, are sketched in Figure 2. The $\mathrm{TE}_{01}$ mode has a circumferential $E_{\phi}$ field, which vanishes at $\rho=0$ and is quite weak at the wall $\rho=a$ (being proportional to $\mathrm{J}_{1}(\mathrm{u} \rho / \mathrm{a})$ ). It follows that the $\mathrm{TE}_{01}$ mode can be excited by a circumferentially oriented linear dipole. For optimum excitation, the linear dipole should neither be at the center or very close to the wall. Alternatively, the $\mathrm{TE}_{01}$ mode can also be excited by a current loop placed in the cross section plane near the center of the tunnel. This will couple with $\mathrm{H}_{z}$ which is maximum at the tunnel center. Dudley (2005) has given rigorous treatment of $\mathrm{TE}_{0}$ modes excitation by a loop, which is located coaxially with the tunnel.

On the other hand, the $\mathrm{HE}_{11}$ mode is almost linearly polarized as demonstrated in the Appendix (see equation A6). So, this mode is optimally excited by a linear dipole close to the center of the tunnel. A detailed rigorous treatment of the $\mathrm{HE}_{\mathrm{nm}}$ mode excitation by a linear dipole is found in (Dudley and Mahmoud, 2006).

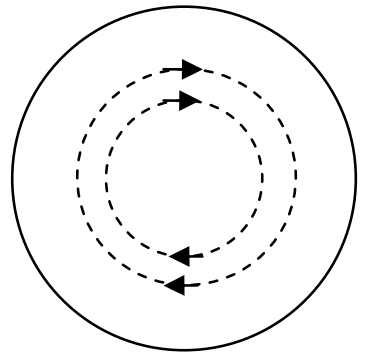

$\mathrm{TE}_{01}$ mode

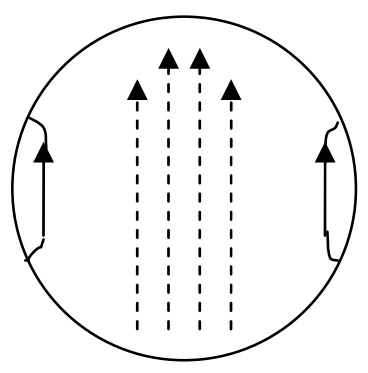

$\mathrm{HE}_{11}$ mode

Fig. 2. E-field lines of the lowest order modes in a circular tunnel.

Here we derive a simple formula for the excitation coefficient of the propagating modes in the tunnel. The source is assumed to be a small linear electric dipole of vector moment ${ }^{\prime} \vec{P}$ ' (Ampere-m) located at $\left(\rho_{1}, \phi_{1}\right)$ in the cross section at, say $\mathrm{z}=0$ and oriented along an arbitrary direction in the transverse plane. The total excited fields $(\vec{E}, \vec{H})$ are expressed as a sum over the natural modes in the tunnel, so

$$
(\vec{E}, \vec{H})=\sum_{r} A_{r}^{ \pm}\left(\vec{e}_{r}, \vec{h}_{r}\right) \exp \left(\mp j \beta_{r} z\right)
$$

Where $\left(\vec{e}_{r}, \vec{h}_{r}\right), r=1,2 \ldots$ are the normal modal fields ordered in an arbitrary manner. The $A_{r}$ are the excitation coefficients. The \pm signs correspond to the fields in the $z>0$ and $z<0$ respectively. To the above sum we should add a continuous of waves representing radiation 
through the surrounding host medium. However, this is normally heavily attenuated as demonstrated in (Dudley and Mahmoud, 2006) and hence will be omitted. The mode excitation coefficients are determined based on the orthogonality among the modes in a tunnel with constant impedance walls (Mahmoud, 1991, section 3.6) and the field discontinuity at the source. So we get ((Mahmoud, 1991, section 2.11).

$$
A_{r}^{+}=A_{r}^{-}=\frac{-\vec{P} \cdot \vec{e}_{r}}{2 \int_{S}\left(\vec{e}_{r} \times \vec{h}_{r}\right) \cdot \hat{z} d s}
$$

where the integration is taken over the tunnel cross section bounded by the walls.

Exercise 3: For each of the linear electric dipoles shown in Figure 3, what are the important excited modes? [HE11 for dipoles 1, and 2, Both HE11 and TE01 modes for dipoles 3 and 4$]$.

Exercise 4: Apply (18) to get the excitation coefficient for the HE11 mode when excited by a unit y-directed dipole moment; $\vec{P}=\hat{y}$ located at the center of the tunnel $\rho=0$. Note that $\left(\vec{e}_{t}, \vec{h}_{t}\right)$ are given by equations (A6-A7) in the Appendix. To simplify the problem,

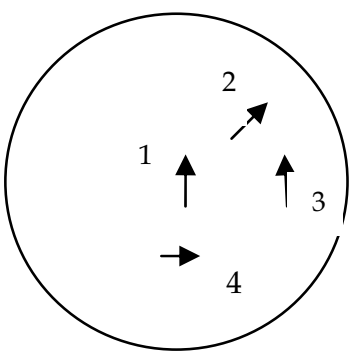

Fig. 3. assume that the frequency is high enough so that $\beta \cong k_{0}$ and $\Lambda \cong+1$. Find the power launched in the $\mathrm{HE}_{11}$ mode.

\section{A rectangular tunnel}

Modal propagation in rectangular waveguides with imperfectly conducting walls is considered to be an intractable problem in waveguide theory. In fact, as indicated by (Wait, 1967) long time ago, there is a fundamental difficulty with mode analysis in cylindrical waveguides with finite impedance walls and cross-sections other than circular. In such waveguides, a natural mode does not take the form of a wave with a single transverse wavenumber as is the case with waveguides with perfectly reflecting walls. In contrast, a waveguide mode is generally composed of a weighted sum of elementary waves having a single transverse wavenumber each. A comprehensive discussion of this phenomenon is reviewed by Mahmoud (1991 sec. 3.5) in relation to elliptical and rectangular waveguides. However, simple, but approximate modal solutions valid in the high frequency regime have been obtained by several authors. Marcatili \&Scmeltzer (1964) obtain the $x$ and $y$ transverse wavenumber approximately by treating the rectangular waveguide as two parallel plates in the $y$ and $x$ directions respectively. Andersen et al. (1975) derive an aprproximate modal equation for the transverse wavenumber by considering the mode to be composed of four crossing plane waves interconnected at the tunnel walls by reflection matrices. The method accounts for the coupling between horizontal and vertical polarizations upon reflection. 
Wait [1980] and Mahmoud [1991, sec. 6.2.2] obtain an approximate TM type modal solution based on the neglect of one weak boundary condition. In the following, we outline this formulation.

So, consider a rectangular guide of width $w$ and height $h$ and outer medium of complex relative permittivity $\varepsilon_{\mathrm{c}}\left(=\varepsilon_{r}-i \sigma / \omega \varepsilon_{0}\right)$ as depicted in Figure 4 . The walls are characterized by constant impedance and admittance $\bar{Z}_{s}, \bar{Y}_{s}$ as given by (4). For a $\mathrm{TM}_{\mathrm{y}}$ mode, or a vertically polarized mode, $E_{x}=0$ and $E_{y}$ may be given, for an even mode, by:

$$
E_{y}=\cos \left(k_{y} y\right) \cos \left(k_{x} x\right) \exp (-j \beta z)
$$

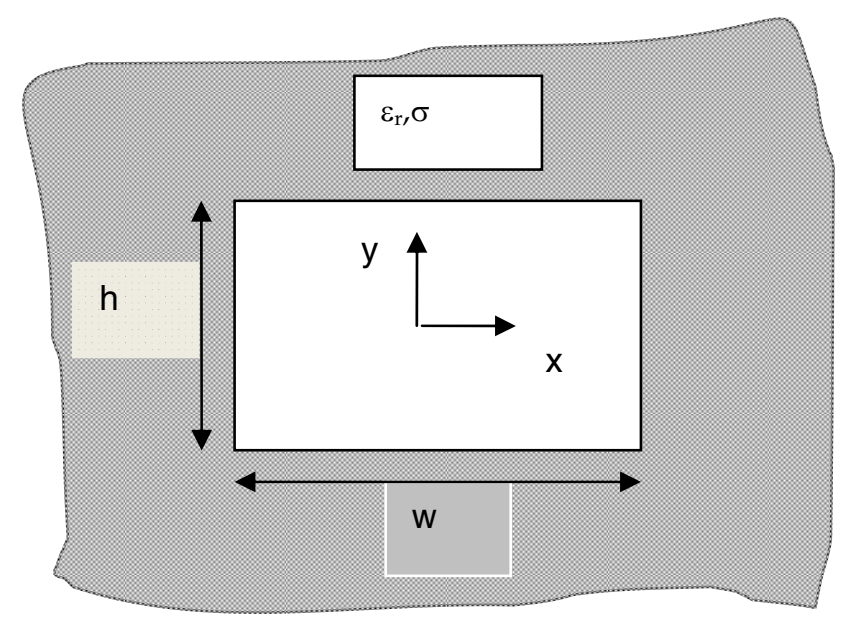

Fig. 4. A rectangular tunnel in a host medium

In the above: $k_{y}^{2}+k_{x}^{2}=k_{0}^{2}-\beta^{2}$. Because the guide is oversized relative to the wavelength, both $k_{x}$ and $k_{y}$ are $<<k_{0}$ and $\beta$ for the low order modes. The $E_{z}$ component is obtained from the divergence equation $\nabla \cdot \vec{E}=0$, hence:

$$
j \beta E_{z}=\partial E_{y} / \partial y
$$

which shows that $E_{z}$ is of first order smallness relative to $E_{y}$. The magnetic field components are obtained as:

$$
\eta_{0} H_{x}=-\beta E_{y} / k_{0}, \eta_{0} H_{y} \approx 0, \text { and } \eta_{0} H_{z}=j \partial E_{y} / k_{0} \partial x
$$

where terms of second order smallness have been neglected (such as $k_{x} k_{y} / k_{0}^{2}$ ). Now the boundary condition at $\mathrm{y}= \pm h / 2$ requires that: $\left.\left(\eta_{0} H_{x} / E_{z}\right)\right|_{y= \pm b}= \pm \bar{Y}_{s}$, which reduces to: 


$$
k_{y} h \tan \left(k_{y} h / 2\right)=j k_{0} h / \bar{Y}_{s}
$$

This is an equation for the y-wavenumber and its solution leads to a set of eigenvalues $k_{y n}$, $\mathrm{n}=1,2 \ldots$ Now we consider the side walls at $x= \pm w / 2$. The boundary conditions at these two walls are

$$
\begin{aligned}
& E_{y}= \pm \eta_{0} \bar{Z}_{s} H_{z} \\
& \eta_{0} H_{y}=\mp \bar{Y}_{s} E_{z}
\end{aligned}
$$

Using (19) and (21) in (23) leads to a modal equation for $k_{x}$.

$$
k_{x} w \tan \left(k_{x} w / 2\right)=j k_{0} w / \bar{Z}_{s}
$$

So (25) is an equation for $k_{x}$ whose solution leads to a set of eigenvalues $k_{x m}$. This completes the modal solution except that we have not satisfied boundary condition (24). Fortunately however, $H_{y}$ is of second order smallness for the lower order modes, hence this boundary condition can be safely neglected.

Approximate solutions of (22) and (25) for $k_{y n}$ and $k_{x m}$ in the high frequency regime, $\left(k_{0} h>>\bar{Y}_{s}, k_{0} w>>\bar{Z}_{s}\right)$ are:

$$
\begin{aligned}
& k_{y n} h=n \pi\left[1+j 2 \bar{Y}_{s} / k_{0} h\right] \\
& k_{x m} w=m \pi\left[1+j 2 \bar{Z}_{s} / k_{0} w\right]
\end{aligned},
$$

where $\mathrm{m}$ and $\mathrm{n}=1,3 \ldots$ are odd integers for the even modes considered. The corresponding mode attenuation rate is easily obtained as:

$$
\alpha_{\mathrm{VPmn}}=2 \pi^{2} n^{2} \operatorname{Re}\left(\bar{Y}_{s}\right) / k_{0}^{2} h^{3}+2 \pi^{2} m^{2} \operatorname{Re}\left(\bar{Z}_{s}\right) / k_{0}^{2} w^{3} \quad \text { Neper } / \mathrm{m}
$$

The attenuation rate of the corresponding horizontally polarized mode may be obtained from (27) by exchanging $w$ and $h$. So:

$$
\alpha_{H P m n}=2 \pi^{2} n^{2} \operatorname{Re}\left(\bar{Y}_{s}\right) / k_{0}^{2} w^{3}+2 \pi^{2} m^{2} \operatorname{Re}\left(\bar{Z}_{s}\right) / k_{0}^{2} h^{3} \quad \text { Neper } / \mathrm{m}
$$

These formulas agree with those derived by Emslie et al (1975). It is worth noting that like the circular tunnel, the attenuation of the dominant modes is inversely proportional to the frequency squared and the linear dimensions cubed. Comparing (27) and (28), we infer that the vertically polarized mode suffers higher attenuation than the horizontally polarized mode for $w>h$. Thus, for a rectangular tunnel with $w>h$, the first horizontally polarized mode; $\mathrm{TM}_{\mathrm{x} 11}$ is the lowest attenuated mode.

Exercise 5: Use (26) to derive (27). In doing so, note that $\alpha=-\operatorname{Im}\left[\left(k_{0}^{2}-k_{x m}^{2}-k_{y n}^{2}\right)^{1 / 2}\right] \cong\left(1 / 2 k_{0}\right) \operatorname{Im}\left[k_{x m}^{2}+k_{y n}^{2}\right]$. This, of course, is valid only for low order modes such that $m \pi / \mathrm{w}$ and $n \pi / h$ are $<<k_{0}$. Compute the attenuation rate of the $\mathrm{TM}_{\mathrm{y} 11}$ and $\mathrm{TM}_{\mathrm{x} 11}$ modes in a tunnel having $\mathrm{w}=2 \mathrm{~h}=4.3$ meters at $1 \mathrm{GHz}$. Take $\varepsilon_{\mathrm{r}}=10$ and $\sigma=0 .[13.27$ and $2.95 \mathrm{~dB} / 100 \mathrm{~m}]$ 
We can infer from the above discussion that the attenuation caused by the walls which are perpendicular to the major electric field is much higher than that contributed by the walls parallel to the electric field.

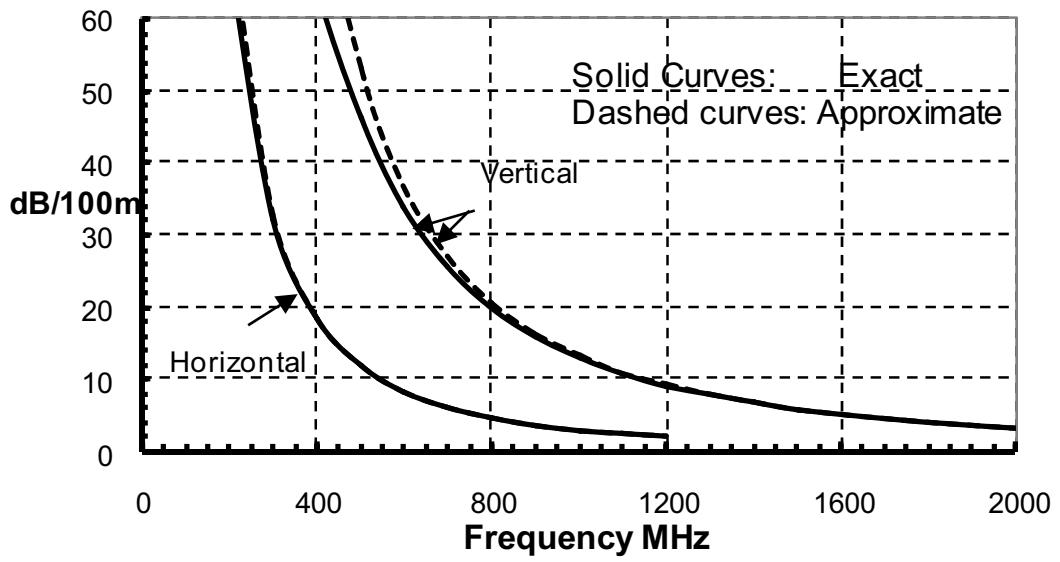

Fig. 5. Attenuation rates in $\mathrm{dB} / 100 \mathrm{~m}$ of $\mathrm{VP}$ and $\mathrm{HP}$ modes with $\mathrm{m}=\mathrm{n}=1$ in a rectangular tunnel of dimensions $4.3 \times 2.15 \mathrm{~m} . \varepsilon_{\mathrm{r}}=10$.

The approximate attenuation rates given by (27-28) for the horizontally and vertically polarized (HP and VP) modes with $m=n=1$ are plotted versus the frequency in Figure 5. Here the tunnel dimensions are chosen as $(w, h)=(4.3 \mathrm{~m}, 2.15 \mathrm{~m})$ and $\varepsilon_{\mathrm{r}}=10$. It is clear that the VP mode has considerably higher attenuation than its HP counterpart. The attenuation rates obtained by exact solution of equations (22) and (25) are also plotted for comparison. It is clear that both solutions coincide at the higher frequencies.

\section{Ray theory:}

When it is required to estimate the field at distances close to the source, the mode series becomes slowly convergent since it is necessary to include many higher order modes. As clear from the above argument, higher order modes are hard to analyze in a rectangular tunnel. In this case the ray series can be adopted for its fast convergence at short distances, say, of tens to few hundred meters from the source. At such distances, the rays are somewhat steeply incident on the walls, hence their reflection coefficients decrease quickly with ray order. Therefore, a small number of rays are needed for convergence.

A geometrical ray approach has been presented by (Mahmoud and Wait 1974a) where the field of a small linear dipole in a rectangular tunnel is obtained as a ray sum over a twodimensional array of images. It is verified that small number of rays converges to the total field at sufficiently short range from the source. Conversely the number of rays required for convergence increase considerably in the far ranges, where only one or two modes give an accurate account of the field. The reader is referred to the above paper for a detailed discussion of ray theory in oversized waveguides. 


\section{Arched Tunnel}

So far we have been studying tunnels with regular cross sections having either circular or rectangular shape. These shapes are amenable to analytical analysis that lead to full characterization of their main modes of propagation. However, most existing tunnels do not have regular cross sections and their study may require exhaustive numerical methods (Pingenot et al., 2006). In this section we consider cylindrical tunnels whose cross-section comprise a circular arch with a flat base as depicted in Figure 6. This can be considered as a circular tunnel whose shape is perturbed into a flat-based tunnel. So, we use the perturbation theory to predict attenuation and phase velocity of the dominant modes from those in a perfectly circular tunnel.

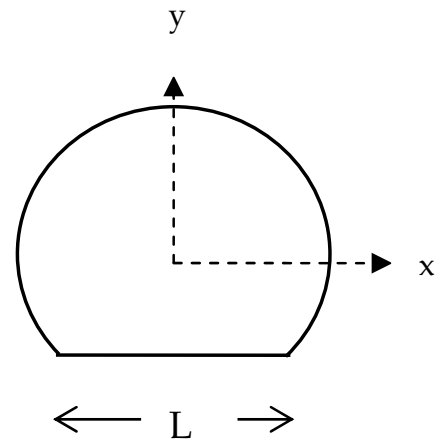

Fig. 6. An arched tunnel with radius $a$ and flat base $L$.

\subsection{Perturbation Analysis}

We consider a cylindrical circular tunnel of radius ' $a$ ' and cross section $S_{0}$ surrounded by a homogeneous earth of relative permittivity $\varepsilon_{r}$. Let us denote the vector fields of a given mode by $\left(\vec{E}_{0}, \vec{H}_{0}\right) \exp \left(-\gamma_{0} z\right)$ where $\gamma_{0}$ is the longitudinal (along $+\mathrm{z}$ ) propagation constant. Similarly, let $(\vec{E}, \vec{H}) \exp (+\gamma z)$ be the vector fields of the corresponding mode in the perturbed tunnel of of area $S$ (Figure 7). Note, however, that the mode is a backward mode; travelling in the $(-z)$ direction. Both circular and perturbed tunnels have the same wall constant impedance $Z$ and admittance $Y$. Now, we use Maxwell's equations that must be satisfied by both modal fields to get the reciprocity relation $\nabla \cdot\left(\vec{E}_{0} \times \vec{H}-\vec{E} \times \vec{H}_{0}\right)=0$. Integrating over the infinitesimal volume between $\mathrm{z}$ and $\mathrm{z}+\mathrm{dz}$ in the perturbed tunnel, we get, after some manipulations, 


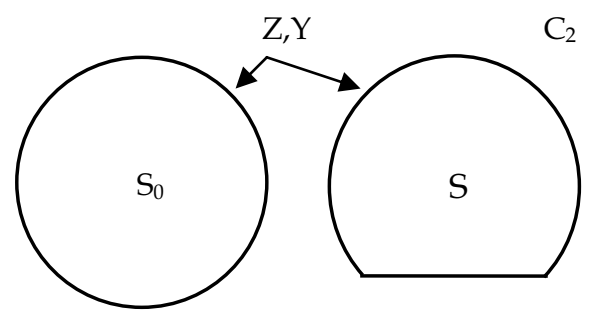

$\mathrm{C}_{1}$

Fig. 7. A circular tunnel and a perturbed circular tunnel with a flat base. The walls are characterized by constant $\mathrm{Z}$ and $\mathrm{Y}$.

$$
\gamma-\gamma_{0}=\frac{-\int_{C 1}\left(\vec{E}_{0} x \vec{H}-\vec{E} x \vec{H}_{0}\right) \cdot \hat{a}_{n} d C_{1}}{\iint_{S}\left(\vec{E}_{0} x \vec{H}-\vec{E} x \vec{H}_{0}\right) \cdot \hat{a}_{z} d S}
$$

where $C_{1}$ is the flat part of the cross- section contour, $\hat{a}_{n}$ is a unit vector along the outward normal to the wall $\left(=-\hat{a}_{y}\right)$ and $\hat{a}_{z}$ is a unit axial vector. The integration in the denominator is taken over the cross section of the perturbed tunnel. In order to evaluate the numerator of (2), we use the constant wall impedance and admittance satisfied by the perturbed fields on the flat surface: $E_{x}=Z_{s} H_{z}$ and $H_{x}=-Y_{s} E_{z} . Z_{\mathrm{s}}$ and $Y_{\mathrm{s}}$ are given in (1-2). Using these relations, (29) reduces to:

$$
\gamma-\gamma_{0}=\frac{-2 \int_{x=0}^{L / 2}\left[E_{0 x} H_{z}+E_{z} H_{0 x}+Y_{s} E_{z} E_{0 z}-Z_{s} H_{z} H_{0 z}\right] d x}{\iint_{S}\left(\vec{E}_{0} x \vec{H}-\vec{E} x \vec{H}_{0}\right) \cdot \hat{a}_{z} d S}
$$

The integration in the numerator is taken over the flat surface of the perturbed tunnel. So far, the above result is rigorous, but cannot be used as such since the perturbed fields are not known. As a first approximation we can equate these fields to the backward mode fields in the un-perturbed (circular) tunnel. So we set: $H_{z} \approx H_{0 z}, E_{z} \approx-E_{0 z}$ in the numerator. In the denominator, the fields involved are the transverse fields (to $\mathrm{z}$ ). So we use the approximations: $\vec{E} \times \hat{a}_{z} \approx \vec{E}_{0} \times \hat{a}_{z}$ and $\vec{H} \times \hat{a}_{z} \approx-\vec{H}_{0} \times \hat{a}_{z}$. Therefore (30) is approximated by:

$$
\gamma-\gamma_{0} \cong \frac{\int_{x=0}^{L / 2}\left[E_{0 x} H_{0 z}-E_{0 z} H_{0 x}-Y_{s} E_{0 z}^{2}-Z_{s} H_{0 z}^{2}\right] d x}{\iint_{S}\left(\vec{E}_{0} \mathrm{x} \vec{H}_{0}\right) \cdot \hat{a}_{z} d S}
$$

Thus, for a given mode in the circular tunnel, (31) can be used to get the propagation constant of the perturbed mode in the corresponding perturbed tunnel. Of particular 
interest is the attenuation factor of the various modes. As a numerical example, we consider an arched tunnel of radius $a=2$ meters with a flat base of width $L$. The surrounding earth has a relative permittivity $\varepsilon_{r}=6$. For an applied frequency $f=500 \mathrm{MHz}$, the modal attenuation factor, computed by (31), is plotted in Figure 8 for the perturbed $\mathrm{TE}_{01}$ and $\mathrm{HE}_{11}$ modes as a function of the $L / a$. Note that $L / a=0$ corresponds to a full circle and $L / a=2$ corresponds to a half circle. Generally the attenuation increases with $L / a$. The $H_{11}$ mode has two versions depending whether the polarization is horizontal (along x) or vertical (along y). Obviously the two modes are degenerate in a perfectly circular tunnel $(L=0)$. However as $L / a$ increases, this degeneracy breaks down in the perturbed tunnel. It is remarkable to see that the attenuation of the horizontally polarized $\mathrm{HE}_{11}$ mode becomes less than that of the vertically polarized mode in the perturbed tunnel. This agrees with measurements made by Molina et al. (2008).

It is interesting to study the effect of changing the frequency or the wall permittivity on the mode attenuation in the perturbed flat based tunnel. Further numerical results (not shown) indicate that the percentage increase of the attenuation relative to that in the circular tunnel is fairly weak on $f$ and $\varepsilon_{r}$. Since the attenuation in an electrically large circular tunnel is inversely proportional to $f^{2}$, so will be the attenuation in the perturbed flat based tunnel.

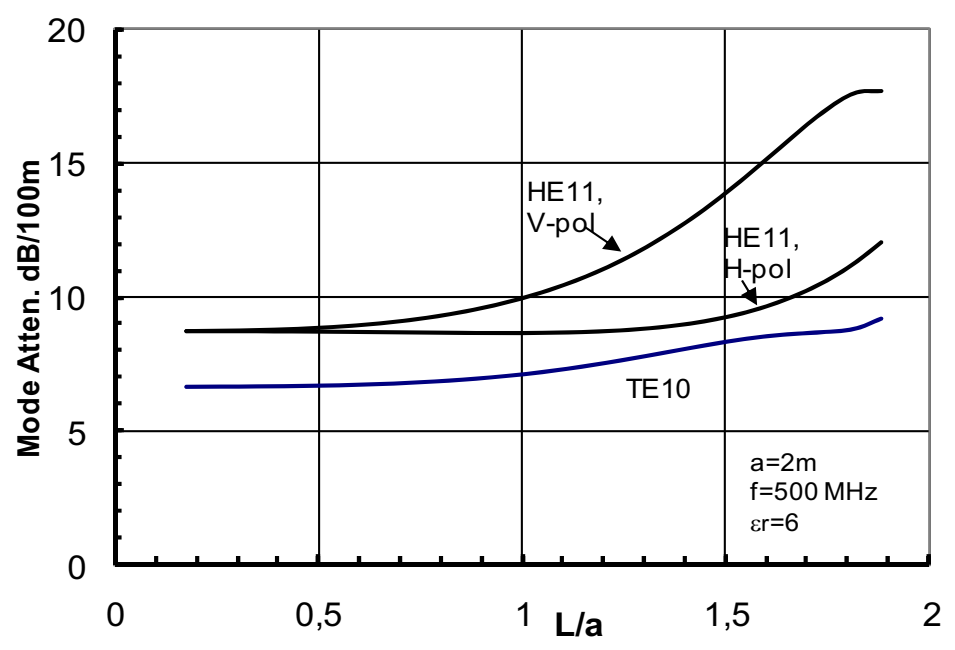

Fig. 8. Attenuation of the perturbed $\mathrm{TE}_{01}$ and $\mathrm{HE}_{11}$ modes in a flat based tunnel versus $L / a$. Note the difference between the attenuation of the VP and the $\mathrm{HP}$ versions of the $\mathrm{HE}_{11}$ mode.

\subsection{An equivalent rectangular tunnel}

As seen in Figure 8, the attenuation of the perturbed $\mathrm{HE}_{11}$ mode in the arched tunnel (with flat base) depends on the mode polarization; namely the vertically polarized $\mathrm{HE}_{11}$ mode is more attenuated than the horizontally polarized mode. The same observation is true for the $\mathrm{HE}_{11}$ mode in a rectangular tunnel whose height ' $h$ ' is less than its width ' $w$ '. This raises the question whether we can model the flat based tunnel of Figure 6 by a rectangular tunnel. We will investigate this possibility in this section. To this end, let us start by comparing the 
attenuation of the $\mathrm{HE}_{\mathrm{nm}}$ mode in tunnels with circular and a square cross sections. For the circular tunnel we have from (15)

$$
\left.\alpha\right|_{H E n m}=\frac{Z_{s} / \eta_{0}+Y_{s} \eta_{0}}{2 k_{0}^{2} a^{3}}\left[x_{n-1, m}\right]^{2}
$$

Where $x_{n-1, m}$ is the $m$ th zero of the Bessel function $J_{\mathrm{n}-1}(x)$. This formula is based on the condition: $k_{0} a \gg x_{n-1, m}$. For the rectangular tunnel with width ' $w$ ' and height ' $h$ ' the attenuation of the $\mathrm{HE}_{\mathrm{nm}}$ mode (with vertical polarization) is given by (27) which is repeated here

$$
\left.\alpha\right|_{H E n m}=\frac{2 \pi^{2}}{k_{0}^{2}}\left[\frac{m^{2} Z_{s} / \eta_{0}}{w^{3}}+\frac{n^{2} Y_{s} \eta_{0}}{h^{3}}\right]
$$

This is valid for electrically large tunnel, or when $k_{0}>>m \pi / w$ and $n \pi / h$. Specializing this result for the $\mathrm{HE}_{11}$ mode in a square tunnel $(w=h$ and $m=n=1)$, we get:

$$
\left.\alpha\right|_{H E 11}=\frac{2 \pi^{2}}{k_{0}^{2} w^{3}}\left[Z_{s} / \eta_{0}+Y_{s} \eta_{0}\right]
$$

Now compare the circular tunnel with the square tunnel for the $\mathrm{HE}_{11}$ mode. From (32) and (33), an equal attenuation occurs when

$$
\mathrm{w}=\left(4 \pi^{2} / 2.4048^{2}\right)^{1 / 3} a=1.897 a
$$

which means that the area of the equivalent square tunnel is equal to 1.145 times the area of the circular tunnel. This contrasts the work of (Dudley et.al, 2007) who adopted an equal area of tunnels. It is important to note that this equivalence is valid only for the $\mathrm{HE}_{11}$ mode in both tunnels; for other modes the attenuation in the circular and the square tunnels are generally not equal.

Now let us turn attention to the arched tunnel with flat base (Figure 6) for which we attempt to find an equivalent rectangular tunnel. We base this equivalence on equal attenuation of the $\mathrm{HE}_{11}$ mode in both tunnels. Let us maintain the ratio of areas as obtained from the square and circular tunnels; namely we fix the ratio of the equivalent rectangular area to the arched tunnel area to 1.145. Meanwhile we choose the ratio $h / w$ equal to the arched tunnel height to its diameter. So, we write:

$$
\begin{aligned}
& w h=1.145\left[(\pi-\theta) a^{2}+(L a / 2) \cos \theta\right], \text { and } \\
& h / w=(1+\cos \theta) / 2
\end{aligned}
$$

where $\theta=\operatorname{Arcsin}(L / 2 a)$ is equal to half the angle subtended by the flat base $\mathrm{L}$ at the center of the circle. 


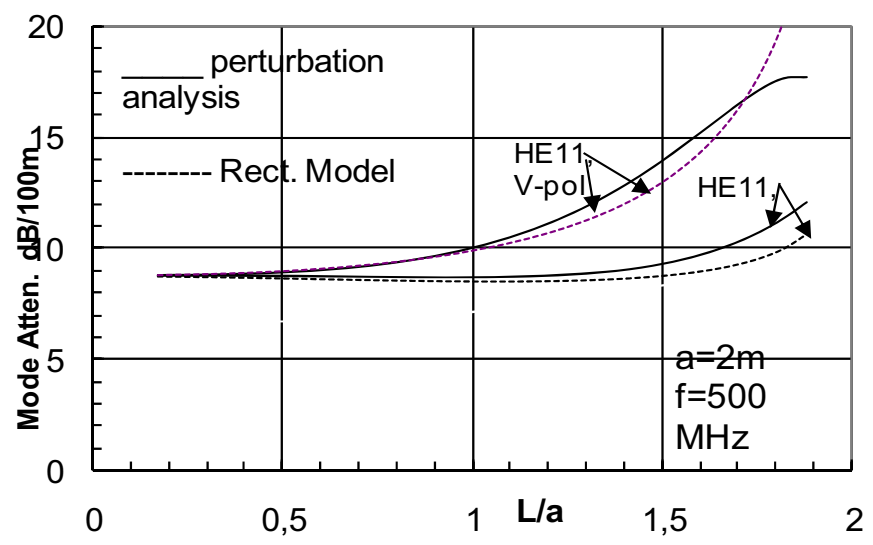

Fig. 9. Attenuation of the $\mathrm{HE}_{11}$ mode in the arched tunnel of Figure 6 using perturbation analysis and rectangular equivalent tunnel

Equation (34) defines the rectangular tunnel that is equivalent to the perturbed circular tunnel regarding the $\mathrm{HE}_{11}$ mode. In order to check the validity of this equivalence, we compare the estimated attenuation of the $\mathrm{HE}_{11}$ mode in the perturbed circular tunnel as obtained by perturbation analysis and by the equivalent rectangular tunnel in Figure 9. There is a reasonably close agreement between both methods of estimation for values of $L / a$ between zero and $\sim 1.82$.

\section{Curved Tunnel}

Modal propagation in a curved rectangular tunnel has been considered by Mahmoud and Wait (1974b) and more recently by Mahmoud (2005). The model used is shown in Figure 10 where the curved surfaces coincide with $\rho=R-w / 2$ and $\rho=R+w / 2$ in a cylindrical frame $(\rho, \phi, z)$ with $z$ parallel to the side walls. The tunnel is curved in the horizontal plane with assumed gentle curvature so that the mean radius of curvature $R$ is $>>w$. The analysis is made in the high frequency regime so that $k_{0} w>>1$. The modes are nearly TE or TM to $z$ with horizontal or vertical polarization respectively. The modal equations for the lower order $T E_{z}$ and $T M_{z}$ modes are derived in terms of the Airy functions and solved numerically for the propagation constant along the $\phi$ - direction. Numerical results are given in (Mahmoud, 2005) and are reproduced here in figures 11 and 12 for the dominant mode with vertical and horizontal polarization respectively. It is seen that wall curvature causes drastic increase of the attenuation especially for the horizontally polarized mode.

This can be explained by noting that the horizontal electric field is perpendicular to the curved walls, causing more attenuation to incur for this polarization.

Further study of the modal fields shows that these fields cling towards the outer curved wall casing increased losses in the wall. Besides, the mode velocity slows down. 


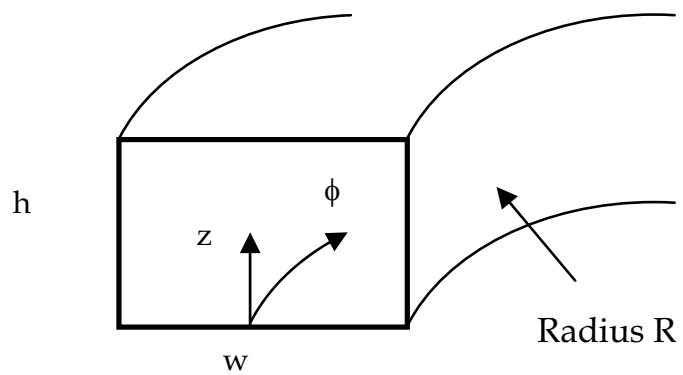

Fig. 10. A curved rectangular tunnel

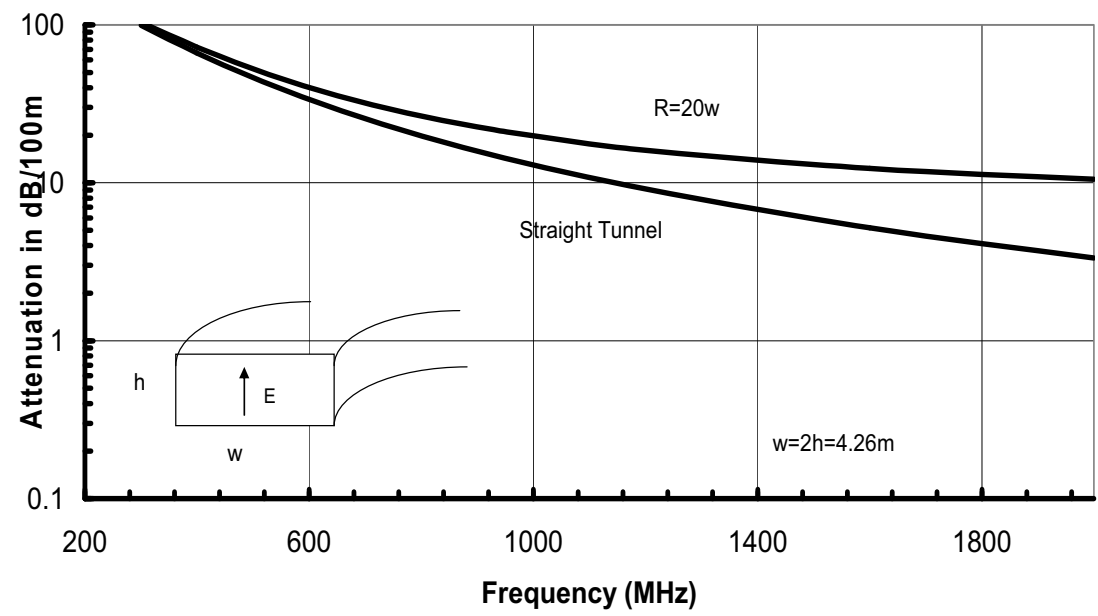

Fig. 11. Attenuation of $\mathrm{TM}_{\mathrm{y} 11}(\mathrm{VP})$ mode in a curved tunnel

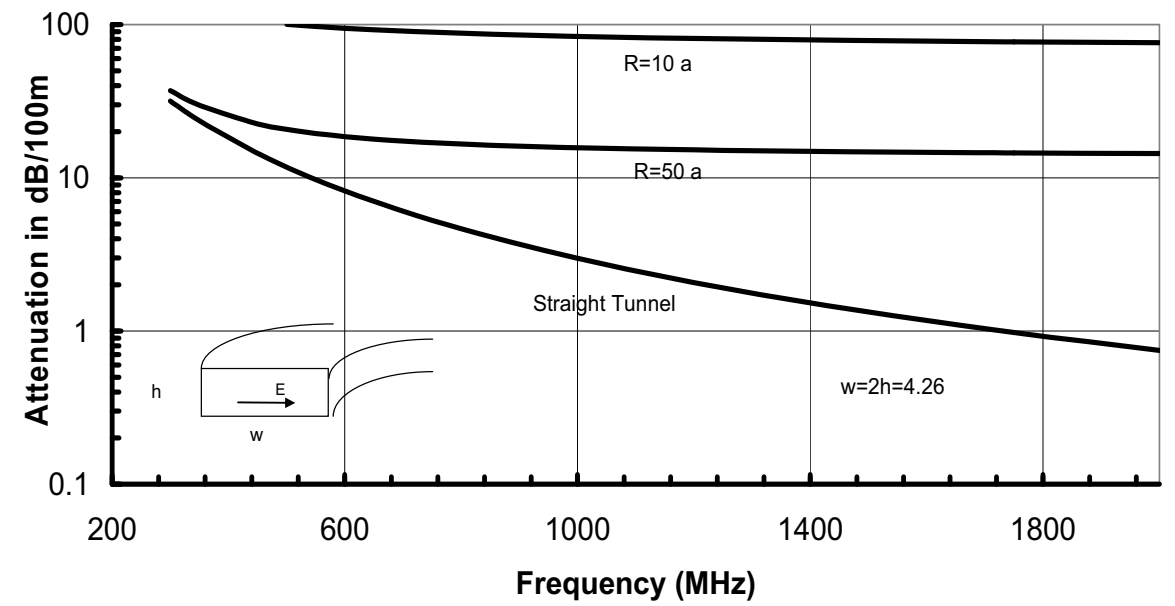

Fig. 12. Attenuation of $\mathrm{TM}_{\mathrm{x} 11}(\mathrm{HP})$ mode in a curved tunnel 


\section{Experimental work}

Measurements of attenuation of the dominant mode in a straight rectangular mine tunnel were given by Goddard (1973). The tunnel cross section was $14 \times 7$ feet (or $4.26 \times 2.13 \mathrm{~m}$ ) and the external medium had $\varepsilon_{r}=10$ and the attenuation was measured at 200, 450 and 1000 MHz. Emslie et al [25] compared these measurements with their theoretical values for the dominant horizontally polarized mode. Good agreement was observed at the first two frequencies, but the experimental values were considerably higher than the theoretical attenuation at the $1000 \mathrm{MHz}$. Similar trend has also been reported more recently by Lienard and Degauque (2000). The difference between the measured and theoretical attenuation at the $1000 \mathrm{MHz}$ was attributed by Emslie et al.(1975) to slight tilt of the tunnel walls. Namely, by using a rather simple theory, it was shown that the increase of attenuation of the dominant mode due to wall tilt is proportional to the frequency and the square of the tilt angle. As a result, it was deduced that the high frequency attenuation of the dominant mode in a rectangular tunnel is governed mainly by the wall tilt.

Goddard (1973) has also measured the signal level around a corner and inside a crossed tunnel. The attenuation rate was very high for a short distance after which the attenuation approaches that of the dominant horizontally polarized mode. Emslie et al. (1975) have explained such behavior as follows. They argue that the crossed tunnel is excited by the higher order modes (or diffused waves in their terms) in the main tunnel. The modes excited in the crossed tunnel are mostly higher order modes with a small component of the dominant mode. These high order modes exhibit very high attenuation for a short distance after which the dominant mode becomes the sole propagating mode. So the signal level starts with a large attenuation rate which gradually decreases towards the attenuation rate of the dominant mode. The theory presented accordingly shows good agreement with measurements. More recently, Lee and Bertoni (2003) evaluated the modal coupling for tunnels or streets with L, T or cross junctions using hybrid ray-mode conversion. They argue that coupling occurs by rays diffracted at the corners into the side tunnels. It is found that the coupling loss is greatest at L-bends and least for cross junctions.

Chiba et.al (1975) have provided field measurements in one of the National Japanese Railway tunnels located in Tohoku. The tunnel cross section is an arch with a flat base as that depicted in Figure 6. The radius $a=4.8 \mathrm{~m}, L=8.8 \mathrm{~m}(L / a=1.83)$, the wall $\varepsilon_{\mathrm{r}}=5.5$ and $\sigma=0.03$ $\mathrm{S} / \mathrm{m}$. Field measurements were taken down the tunnel for different frequencies and polarizations. The attenuation of the dominant $\mathrm{HE}_{11}$ mode was then measured for both horizontal and vertical polarization at the frequencies 150, 470, 900, 1700, and $4000 \mathrm{MHz}$. We plot the predicted attenuation of the horizontally polarized $\mathrm{HE}_{11}$ mode in this same tunnel using both the perturbation analysis and the rectangular tunnel model in Figure 13. On top of these curves, the measured attenuation is shown as discrete dots at the above selected frequencies. The predicted attenuation shows the expected inverse frequency squared dependence. The measured attenuation follows the predicted attenuation except at the highest two frequencies (1700 and $4000 \mathrm{MHz}$ ) whence it is higher than predicted. This can be explained on account of wall roughness or micro-bending of the tunnel walls that affect the higher frequencies in particular. 


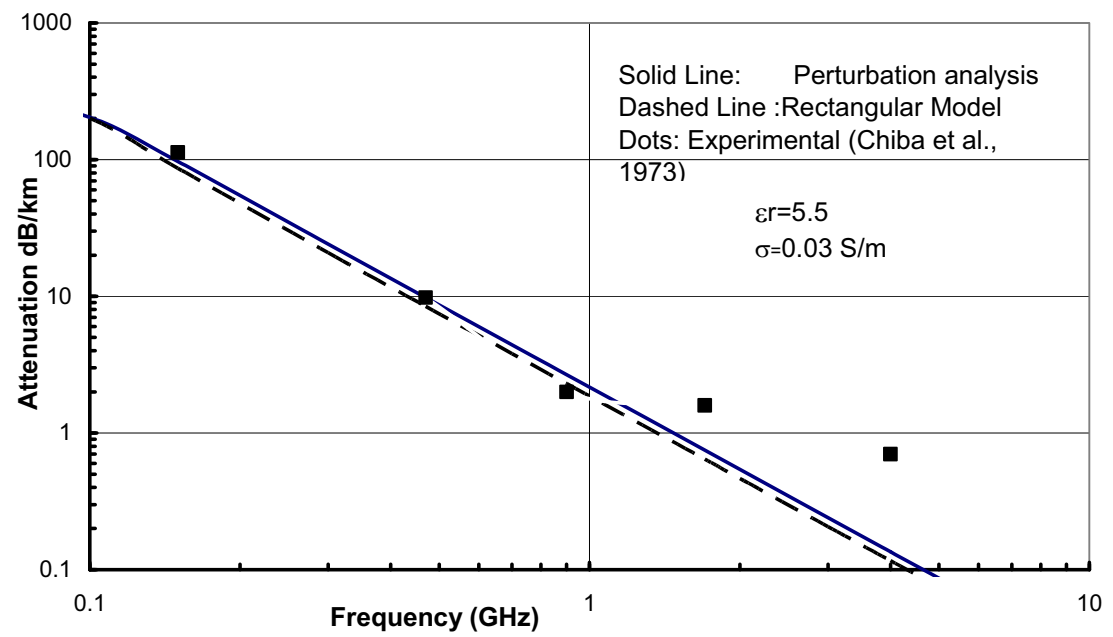

Fig. 13. Attenuation of the $\mathrm{HE}_{11}$ mode in the Japanese National Ralway tunnel by the perturbation analysis and the rectangular tunnel model versus measured values (as reported in (Chiba et al., 1973).

Measurements of the electric field down the Massif Central road tunnel south Central France have been taken by the research group in Lille University and the results are reported by Dudley et.al. (2007). The Massif Central tunnel has a flat based circular arch shape as in Figure 6 with radius $a=4.3 \mathrm{~m}$ and $L=7.8 \mathrm{~m}$; that is $L / a=1.81$. The relative permittivity of the wall $\varepsilon_{\mathrm{r}}=5$ and the conductivity $\sigma=0.01 \mathrm{~S} / \mathrm{m}$. The transmit and receive antennas were vertically polarized and the field measured down the tunnel at the frequencies 450 and $900 \mathrm{MHz}$ are given in Figure 14. For the lower frequency, the field shows fast oscillatory behavior in the near zone, but at far distances from the source (greater than $\sim 1800 \mathrm{~m}$ ), the field exhibits almost a constant rate of attenuation, which is that of the dominant $\mathrm{HE}_{11}$ (like) mode. We estimate the attenuation of this mode as $27.2 \mathrm{~dB} / \mathrm{km}$. At the $900 \mathrm{MHz}$ frequency, there are two interfering modes that are observed in the range of 1500$2500 \mathrm{~m}$. One of these two modes must be the dominant $\mathrm{HE}_{11}$ mode. Some analysis is needed in this range that lead to an estimation of the attenuation of the $\mathrm{HE}_{11}$ mode, which we find as $6.8 \mathrm{~dB} / \mathrm{km}$. 


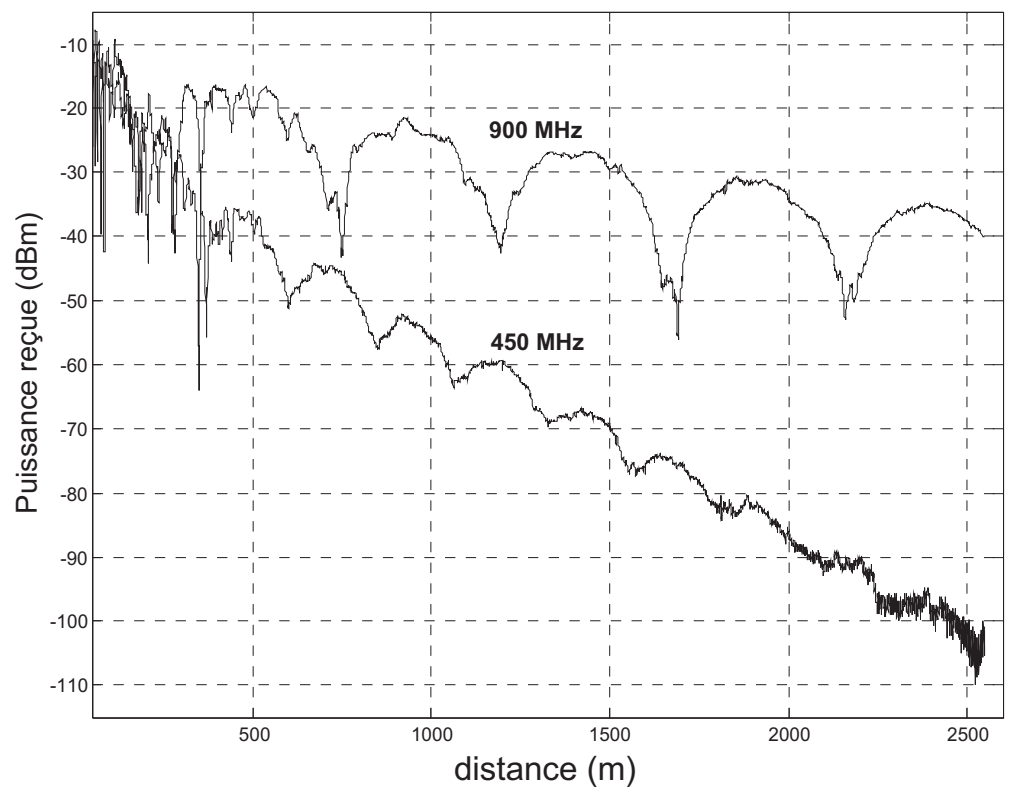

Fig. 14. Measured field down the Massif Central Tunnel in South France (Dudley et al., 2007) at 450 and $900 \mathrm{MHz}$.

A comparison between these measured attenuation rates and those predicted by the perturbation analysis or the equivalent rectangular tunnel (given in section 5) is made in Table 2. Good agreement is seen between predicted and measured attenuation although the measured values are slightly higher. This can be attributed to wall roughness and microbending.

\begin{tabular}{|c|c|c|c|}
\hline & $\begin{array}{c}\text { Perturbation } \\
\text { Analysis }(\mathrm{dB} / \mathrm{km})\end{array}$ & $\begin{array}{c}\text { Equivalent } \\
\text { Rectangular model }\end{array}$ & $\begin{array}{c}\text { Measured } \\
\text { Attenuation }\end{array}$ \\
\hline$f=450 \mathrm{MHz}$ & 22.0 & 24.1 & 27.2 \\
\hline$f=900 \mathrm{MHz}$ & 5.52 & 6.03 & 6.8 \\
\hline
\end{tabular}

Table 2. Measured versus predicted attenuation rates of the $\mathrm{HE}_{11}$ mode in the Massif Central Road Tunnel, South France.

\section{Concluding discussion}

We have presented an account of wireless transmission of electromagnetic waves in mine and road tunnels. Such tunnels act as oversized waveguides to UHF and the upper VHF waves. The theory of mode propagation in straight tunnels of circular, rectangular and arched cross sections has been covered and it is demonstrated that the dominant modes attenuate with rates that decrease with the applied frequency squared. We have also studied the increase of 
mode attenuation caused by tunnel curvature. Comparison of the theory with existing experimental measurements in real tunnels show good agreement except at the higher frequencies at which wall roughness, and microbending can increase signal loss over that predicted by the theory. While the higher order modes are highly attenuated and therefore contribute to signal loss, they can be beneficial in allowing the use of Multiple Input - Multiple Output (MIMO) technique to increase the channel capacity of tunnels. A detailed account of this important topic is found in (Lienard et al, 2003) and (Molina et al., 2008).

\section{References}

Andersen, J.B.; Berntsen, S. \& Dalsgaard, P. (1975). Propagation in rectangular waveguides with arbitrary internal and external media, IEEE Transaction on Microwave Theory and Technique, MTT-23, No. 7, pp. 555-560.

Chiba, J.; Sato, J.R.; Inaba, T; Kuwamoto, Y.; Banno, O. \& Sato, R. “ Radio communication in tunnels", IEEE Transaction on Microwave Theory and Technique, MTT-26, No. 6, June 1978.

Dudley, D.G. (2004). Wireless Propagation in Circular Tunnels, IEEE Transaction on Antennas and Propagation, Vol. 53, n0.1, pp. 435-441.

Dudley, D.G. \& Mahmoud, S.F. (2006). Linear source in a circular tunnel, IEEE Transaction on Antennas and Propagation, Vol. 54, n0.7, pp. 2034-2048.

Dudley, D.G., Martine Lienard, Samir F. Mahmoud and Pierre Degauque, (2007) “Wireless Propagation in Tunnels", IEEE Antenna and Propagation magazine, Vol. 49, no. 2, pp. 11-26, April 2007.

Emslie, A.G.; Lagace, R.L. \& Strong, P.F. (1973). Theory of the propagation of UHF radio waves in coal mine tunnels, Proc. Through the Earth Electromagnetics Workshop, Colorado School of mines, Golden, Colorado, Aug. 15-17.

Emslie, A.G.; Lagace R.L. \& Strong, P.F. (1975). Theory of the propagation of UHF radio waves in coal mine tunnels, IEEE Transaction on Antenn. Propagat., Vol. AP-23, No. 2, pp. 192-205.

Glaser, J.I. (1967). Low loss waves in hollow dielectric tubes, Ph.D. Thesis, M.I.T.

Glaser, J.I. (1969). Attenuation and guidance of modes in hollow dielectric waveguides, IEEE Trans. Microwave Theory and Tech, Vol. MTT-17, pp.173-174.

Goddard, A.E. (1973). Radio propagation measurements in coal mines at UHF and VHF, Proc. Through the Earth Electromagnetics Workshop, Colorado School of mines, Golden, Colorado, Aug. 15-17.

Lee, J. \& Bertoni, H.L. (2003). Coupling at cross, T and L junction in tunnels and urban street canyons, IEEE Transaction on Antenn. Propagat., Vol. AP-51, No. 5, pp. 192-205, pp.926-935.

Lienard, M. \& Degauque, P. (2000). Natural wave propagation in mine environment, IEEE Transaction on Antennas \& propagate, Vol-AP-48, No.9, pp.1326-1339.

Lienard, M.; Degauque, P.; Baudet, J. \& Degardin, D. (2003). Investigation on MIMO Channels in Subway Tunnels, IEEE Journal on Selected Areas in Communication, Vol. 21, No. 3, pp.332-339.

Mahmoud, S.F. \& Wait, J.R. (1974a). Geometrical optical approach for electromagnetic wave propagation in rectangular mine tunnels, Radio Science, Vol. 9, no. 12, pp. 11471158. 
Mahmoud, S.F. \& Wait, J.R. (1974a). Guided electromagnetic waves in a curved rectangular mine tunnel, Radio Science, May 1974, pp. 567-572.

Mahmoud, S.F. (1991) Electromagnetic Waveguides; theory and applications, IEE electromagnetic waves series 32, Peter peregrinus Ltd. on behalf of IEE.

Mahmoud, S.F. (2005). Modal propagation of high frequency e.m. waves in straight and curved tunnels within the earth, Journal of electromagnetic wave applications (JEMWA), Vol. 19, No. 12, pp. 1611-1627.

Marcatili, E.A.J. \& Schmeltzer, R.A. (1964). Hollow metallic and dielectric waveguides for long distsnce optical transmission and lasers, The Bell System Technical Journal, Vol. 43, pp. 1783-1809, July 1964.

Molina-Garcia-Padro, J.M.; Lienard, M. , Nasr, A. \& Degauque, P. (2008). On the possibility of interpreting field variations and polarization in arched tunnels using a model of propagation in rectangular or circular tunnels, IEEE Transaction on Antenn. Propagat., Vol. AP-56, No. 4, pp. 1206-1211.

Molina-Garcia-Pardo, J. M.; Liénard, M.; Degauque, P.; Dudley, D.G. \& and Juan-Llacer, M.(2008). MIMO Channel Characteristics in Rectangular Tunnels from Modal Theory, IEEE Transactions on Vehicular Technology, Vol 57, No. 3, pp.1974-1979.

Pingenot, J.; Rieben, R.N.; White, D.A.; \& Dudley, D.G. (2006). Full wave analysis of RF signal attenuation in a lossy rough surface cave using high order Time Domain vector Finite Element method, Journal of Electromagnetic Wave Applications, Vol.20, no. 12 , pp. 1695-1705.

Stratton, J.A. (1941). Electromagnetic Theory, McGraw Hill, New York.

Wait, J.R (1967). A fundamental difficulty in the analysis of cylindrical waveguides with impedance walls, Electronic Letters, Vol. 3, No. 2, pp. 87-88.

Wait, J.R. (1980). Propagation in rectangular tunnel with imperfectly conducting walls, Electronic Letters, Vol. 16, No. 13, pp. 521-522.

\section{Appendix}

The transverse fields of a hybrid mode in a circular tunnel are obtained from the axial fields by (Mahmoud, 1991, p. 7):

$$
\begin{aligned}
& \vec{E}_{t}=\frac{-j \beta}{k_{\rho}^{2}} \nabla_{t} E_{z}-\frac{j \omega \mu_{0}}{k_{\rho}^{2}} \nabla_{t} E_{z} \mathrm{x} \hat{z} \\
& \vec{H}_{t}=\frac{-j \beta}{k_{\rho}^{2}} \nabla_{t} H_{z}-\frac{j \omega \varepsilon_{0}}{k_{\rho}^{2}} \hat{\mathrm{z}} \mathrm{x} \nabla_{t} E_{z}
\end{aligned}
$$

Where $\nabla_{t}$ is the transverse differential operator: $\nabla_{t}=(\partial / \partial \rho) \hat{\rho}+(\partial / \rho \partial \varphi) \hat{\varphi}$.

Using the axial fields in (5) we get the explicit expressions:

$$
\begin{aligned}
& E_{\rho}=\frac{k_{0}}{k_{\rho}}\left[\left(\beta / k_{0}\right) J_{n}^{\prime}\left(k_{\rho} \rho\right)+\Lambda \frac{n J_{n}\left(k_{\rho} \rho\right)}{k_{\rho} \rho}\right] \sin n \varphi e^{-j \beta z} \\
& E_{\varphi}=\frac{k_{0}}{k_{\rho}}\left[\left(\beta / k_{0}\right) \frac{n J_{n}\left(k_{\rho} \rho\right)}{k_{\rho} \rho}+\Lambda J_{n}^{\prime}\left(k_{\rho} \rho\right)\right] \cos n \varphi e^{-j \beta z}
\end{aligned}
$$


And

$$
\begin{aligned}
& \eta_{0} H_{\rho}=\frac{k_{0}}{k_{\rho}}\left[-\left(\beta \Lambda / k_{0}\right) J_{n}^{\prime}\left(k_{\rho} \rho\right)-\frac{n J_{n}\left(k_{\rho} \rho\right)}{k_{\rho} \rho}\right] \cos n \varphi e^{-j \beta z} \\
& \eta_{0} H_{\varphi}=\frac{k_{0}}{k_{\rho}}\left[\left(\beta \Lambda / k_{0}\right) \frac{n J_{n}\left(k_{\rho} \rho\right)}{k_{\rho} \rho}+J_{n}^{\prime}\left(k_{\rho} \rho\right)\right] \sin n \varphi e^{-j \beta z}
\end{aligned}
$$

It is useful to express the transverse field in terms of Cartesian coordinates $(x, y)$. Using the Bessel function identities:

$$
\begin{aligned}
& n J_{n}(u) / u=\left(J_{n-1}(u)+J_{n+1}(u)\right) / 2 \\
& J_{n}^{\prime}(u)=\left(J_{n-1}(u)-J_{n+1}(u)\right) / 2
\end{aligned}
$$

we get:

$$
\begin{aligned}
& \frac{2 k_{\rho}}{k_{0}} E_{x}=\left[\beta / k_{0}+\Lambda\right] J_{n-1}\left(k_{\rho} \rho\right) \sin (n-1) \varphi+\left[-\beta / k_{0}+\Lambda\right] J_{n+1}\left(k_{\rho} \rho\right) \sin (n+1) \varphi \\
& \frac{2 k_{\rho}}{k_{0}} E_{y}=\left[\beta / k_{0}+\Lambda\right] J_{n-1}\left(k_{\rho} \rho\right) \cos (n-1) \varphi+\left[\beta / k_{0}-\Lambda\right] J_{n+1}\left(k_{\rho} \rho\right) \cos (n+1) \varphi
\end{aligned}
$$

In the special case $n=1,(\mathrm{~A} 5)$ reduces to:

$$
\begin{gathered}
\frac{2 k_{\rho}}{k_{0}} E_{x}=\left[-\beta / k_{0}+\Lambda\right] J_{2}\left(k_{\rho} \rho\right) \sin 2 \varphi \\
\frac{2 k_{\rho}}{k_{0}} E_{y}=\left[\beta / k_{0}+\Lambda\right] J_{0}\left(k_{\rho} \rho\right)+\left[\beta / k_{0}-\Lambda\right] J_{2}\left(k_{\rho} \rho\right) \cos 2 \varphi
\end{gathered}
$$

This shows that for the $\mathrm{HE}_{11}$ mode $(\Lambda \sim+1)$, the field is almost y-polarized. The corresponding magnetic field is

$$
\begin{gathered}
\frac{2 k_{\rho}}{k_{0}} H_{y}=\left[\beta \Lambda / k_{0}-1\right] J_{2}\left(k_{\rho} \rho\right) \sin 2 \varphi \\
\frac{2 k_{\rho}}{k_{0}} H_{x}=-\left[\beta \Lambda / k_{0}+1\right] J_{0}\left(k_{\rho} \rho\right)+\left[\beta \Lambda / k_{0}-1\right] J_{2}\left(k_{\rho} \rho\right) \cos 2 \varphi
\end{gathered}
$$

Equations (A6-A7) give the transverse fields of the $\mathrm{HE}_{1 \mathrm{~m}}$ modes. 


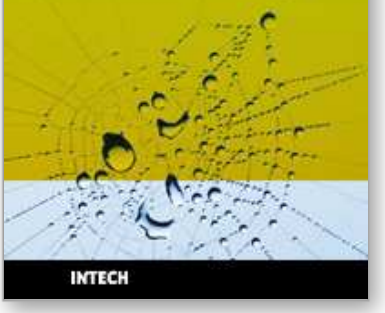

\section{Mobile and Wireless Communications Physical Layer Development and Implementatiom}

Edited by Salma Ait Fares and Fumiyuki Adachi

ISBN 978-953-307-043-8

Hard cover, 290 pages

Publisher InTech

Published online 01, January, 2010

Published in print edition January, 2010

Mobile and Wireless Communications have been one of the major revolutions of the late twentieth century. We are witnessing a very fast growth in these technologies where mobile and wireless communications have become so ubiquitous in our society and indispensable for our daily lives. The relentless demand for higher data rates with better quality of services to comply with state-of-the art applications has revolutionized the wireless communication field and led to the emergence of new technologies such as Bluetooth, WiFi, Wimax, Ultra wideband, OFDMA. Moreover, the market tendency confirms that this revolution is not ready to stop in the foreseen future. Mobile and wireless communications applications cover diverse areas including entertainment, industrialist, biomedical, medicine, safety and security, and others, which definitely are improving our daily life. Wireless communication network is a multidisciplinary field addressing different aspects raging from theoretical analysis, system architecture design, and hardware and software implementations. While different new applications are requiring higher data rates and better quality of service and prolonging the mobile battery life, new development and advanced research studies and systems and circuits designs are necessary to keep pace with the market requirements. This book covers the most advanced research and development topics in mobile and wireless communication networks. It is divided into two parts with a total of thirty-four stand-alone chapters covering various areas of wireless communications of special topics including: physical layer and network layer, access methods and scheduling, techniques and technologies, antenna and amplifier design, integrated circuit design, applications and systems. These chapters present advanced novel and cutting-edge results and development related to wireless communication offering the readers the opportunity to enrich their knowledge in specific topics as well as to explore the whole field of rapidly emerging mobile and wireless networks. We hope that this book will be useful for students, researchers and practitioners in their research studies.

\section{How to reference}

In order to correctly reference this scholarly work, feel free to copy and paste the following:

Samir F. Mahmoud (2010). Wireless Transmission in Tunnels, Mobile and Wireless Communications Physical Layer Development and Implementatiom, Salma Ait Fares and Fumiyuki Adachi (Ed.), ISBN: 978-953-307043-8, InTech, Available from: http://www.intechopen.com/books/mobile-and-wireless-communicationsphysical-layer-development-and-implementatiom/wireless-transmission-in-tunnels

\section{INTECH}


University Campus STeP Ri Slavka Krautzeka 83/A

51000 Rijeka, Croatia

Phone: +385 (51) 770447

Fax: +385 (51) 686166

www.intechopen.com
Unit 405, Office Block, Hotel Equatorial Shanghai No.65, Yan An Road (West), Shanghai, 200040, China 中国上海市延安西路65号上海国际贵都大饭店办公楼 405 单元

Phone: +86-21-62489820

Fax: +86-21-62489821 
(C) 2010 The Author(s). Licensee IntechOpen. This chapter is distributed under the terms of the Creative Commons Attribution-NonCommercial-ShareAlike-3.0 License, which permits use, distribution and reproduction for non-commercial purposes, provided the original is properly cited and derivative works building on this content are distributed under the same license. 